\title{
Consensus on peri-operative surgical practice during the COVID-19 pandemic: an appraisal of the literature
}

\author{
Ravindri Jayasinghe, Umesh Jayarajah, Sanjeewa Seneviratne \\ Department of Surgery, Faculty of Medicine, University of Colombo, Sri Lanka
}

Keywords: Surgery; surgical practice; COVID-19; pandemic; SARS-CoV-2; review

\begin{abstract}
The coronavirus disease-2019 [COVID-19] pandemic is an ongoing pandemic caused by severe acute respiratory syndrome-coronavirus-2 [SARS-CoV-2]. Although COVID19 pandemic is not a direct surgical problem, its impact on the surgical units has been substantial. Many operations have been cancelled or deferred due to the risk of disease spread and staff shortages. Operating theatres are identified as areas of the high risk of disease transmission due to aerosol generation during the surgical procedures and prolonged patient contact. Resource limitations, including lack of personal protective equipment [PPE] and limited availability of testing, continue to expose the surgical community to COVID-19. In this review, evidence and consensus guidelines on surgical practice during the COVID-19 pandemic are summarised and described. Immediate action is deemed essential to ensure the unhindered provision of surgical care while optimizing the use of limited resources and ensuring staff safety.
\end{abstract}

\section{Introduction and Background}

In late December 2019 hospitals in Wuhan, China reported an outbreak of pneumonia of an unknown cause [1]. Later in January 2020, a novel coronavirus strain [SARS-CoV-2] was isolated from the suspected patients. Despite the early recognition and the public health measures, the infection spread rapidly across China and then the rest of the world. The World Health Organization [WHO] declared the outbreak as a Public Health Emergency of International Concern [PHEIC] on January 30th, 2020 [2].

Although COVID-19 is not a primary surgical problem, its impact on the surgical units has been considerable, which to some extent could be viewed as a mass casualty incident with a global impact. With human and financial resources overwhelmed, the rational use of available surgical resources

Correspondence: Umesh Jayarajah

E-mail: umeshe.jaya@gmail.com

(iD) https://orcid.org/0000-0002-0398-5197

Received: 06-08-2020 Accepted: 24-08-202

DOI: http://doi.org/10.4038/sljs.v38i2.8738 has become a matter of utmost importance. The main threats of COVID-19 to the surgical community are related to; staff management, halting elective procedures, effective triaging of overwhelmed resources, practising precautions to combat the high risk of transmission and social distancing at the workplace.

The objective of this review is to summarize and describe the current evidence and consensus guidelines on surgical practice during the COVID-19 pandemic.

\section{Preoperative preparation and general precautions}

Managing logistics to provide continuous optimum patient care should be the goal in all surgical centres. In scheduling patients for surgery, evaluating the individual patient's risks of infection versus consequences due to delay in the procedure should be considered. During the decision making, it is also important to consider the availability of resources such as personal protective equipment [PPE], staff and other medical and logistical concerns [3]. The Royal College of Surgeons of England recommends considering the logistical capacity determined by the theatre manager, the risk to patient outcomes due to the delay determined by a surgeon in the relevant speciality balanced with the capacity of the institution to manage an outbreak. This triage should be based on a real-time data-driven assessment [4].

Wearing adequate PPE should be considered in all situations involving patients confirmed or suspected to have COVID19. The American College of Surgeons has published a set of guidelines on the use of PPE in the operating room [OR] in line with the Centres for Disease Control and Prevention [CDC] recommendations for the use of PPE for healthcare personnel $[5,6]$. In addition to asymptomatic carriers of COVID-19, as the incubation period of the virus may be as long as 14 days, asymptomatic infected individuals also may pose a threat to health care personnel. It is recommended to wear N95 respirators in the OR since standard surgical facemasks are inadequate to protect against virus transmission from aerosol-generating procedures. Depending on the logistic constraints, institutions may implement policies on extended use or limited reuse of N95 respirators to ensure adequate supplies at times of peak demand. Such limitations may prioritize the personnel having the highest 
risk of exposure as recommended by the $\mathrm{CDC}$ and the American Society of Anaesthesiologists [6,7].

The Secretary-General of the WHO emphasized on the importance of testing during the pandemic where he said "You cannot fight a fire blindfolded. Our key message is test, test, test" [8]. Therefore, it is important to consider the preoperative testing of patients undergoing surgery. Priority needs to be given to patients having suspicious symptoms, evidence of respiratory illness, high risk due to contact history and in patients from areas of outbreaks. Although surgeons are encouraged to request testing in patients scheduled for surgery, this must be balanced with the capacity for COVID19 testing and potential risk of COVID-19 in the community.

The available tests for COVID-19 include nucleic acid amplification tests for viral RNA using reverse transcriptionpolymerase chain reaction [RT-PCR] and serology for antibody detection which includes detection of IgM, IgA, IgG or total antibodies [9]. Studies have suggested that most COVID-19 patients seroconvert within seven to eleven days although, some may develop antibodies sooner. Antibody testing is used to identify individuals who were previously infected and recovered as well as individuals who may be immune to COVID-19 [9].

Hospital environment itself may spread the disease to patients awaiting surgery, who in turn may spread the disease among other patients and healthcare staff. This risk will be greater among patients with prolonged hospital stays due to potential cancellations leading to delays in surgery. Therefore, ideally, all patients should be screened with the gold standard test [RTPCR] 24-hours before the procedure with or without an antibody test, followed by isolation until surgery [9]. If they test positive for PCR, public health protocols for COVID-19 should be followed and the surgery postponed in case of routine surgeries. If positive for only the antibodies, they will require further testing to confirm complete recovery from COVID-19. If negative for both the PCR and the antibody screen, still they may require weekly PCR during the hospital stay and be discharged after undergoing a PCR for confirmation [9]. However, such repeated investigations would be a major burden to many resource-limited settings in developing as well as developed countries during the height of the pandemic.

Forrester et al, have recommended assuming all patients undergoing emergency procedures to be infected and appropriate safety precautions be practised. All aerosolgenerating procedures of the upper aero-digestive tract as well as endoscopy, open or laparoscopic bowel surgery are included into the high-risk category, given that the virus may survive in the gastrointestinal tract $[10,11]$. As aerosolization of the virus may occur through exhaled gases, it is advised that even when a procedure is performed under deep sedation, to avoid the use of high flow oxygen and, if feasible the patient to wear a surgical face mask throughout the procedure [12]. When a patient is proven to be positive, after the approval of the surgical and the anaesthetic teams, the surgery could proceed with precautionary measures with all members of the teams wearing appropriate protective attire as recommended by the CDC. For patients who test negative by RT-PCR, the team members may use standard surgical attire $[6,10]$.

Practising social distancing and avoiding public gatherings at the workplace should also be practised as recommended by the CDC to contain the possible spread of the virus within the working environment [13]. Segregating staff between hospitals is an important measure which would also prevent cross-infection among hospitals. Resorting to webconferencing instead of large meetings is recommended and should be implemented. Recording attendance at small group staff meetings would further help contact tracing in the event of an outbreak [14]. Furthermore, it is important to develop a pandemic preparedness protocol in collaboration with surgeons and anaesthesiologists with the involvement of infection control experts which should be incorporated into the routine hospital management plan. Staff training should be considered an immediate priority with effective communication between the hospital's central command team and all surgical teams [15]. In addition to these, Brindle et al had suggested having a clear plan in place for essential surgeries, limiting staff to only the essential number, and having a dedicated operating area for suspected COVID-19 patients [16].

\section{Theatre setting during the COVID-19 pandemic}

Due to the highly contagious nature of the virus and its spread through fomites, it is necessary to have designated ORs for COVID-19 patients undergoing surgery. Choosing an OR near the entrance of the theatre block would minimize the potential for contamination of other theatres [17]. Patient transport to the theatre block is recognized to be associated with a high risk of disease transmission. It is recommended to have a short pre-determined path for transport, avoiding any public areas and areas with non-infected patients as much as possible. The personnel transporting the patient should be equipped with adequate PPE and should have had training in handling high-risk patients. Furthermore, the patient should wear a facemask ideally an N-95 as a precaution. The environmental surfaces coming in contact should be immediately sanitized before and after patient transport. It is useful to have a dedicated team to handle sanitization. Having the same personnel handle a single patient will be beneficial in effectively managing resources. Furthermore, bringing nonessential items to the OR should be avoided. All disposable 
items should be discarded after a single case, traffic in and out of the theatre should be minimised, a dedicated recovery area should be allocated for suspected patients, or patients may be recovered within the OR itself [16]. Where possible, the duration of the case could be shortened to decrease staff exposure [16].

Standard ORs are designed to have a positive pressure environment. However, during the pandemic, it is ideal to have a negative pressure environment with a high $[25$ per hour] frequency of air changes which will minimize dissemination of the virus from the OR. Measures should be taken to limit the flow of contaminated air out of the OR through only one outlet. A designated workflow should also be established with coordination of the staff while allocating a separate anesthesiologist for the designated OR with the allocation of a separate area for donning and doffing of PPE as recommended by the CDC [18]. Furthermore, strict infection control practices and decontamination procedures including routine checks and cleaning of the anaesthetic machines and powered air-purifying respirator [PAPR] sets are necessary [14]. Where feasible, it is advised to discard the anaesthetic breathing circuit and the canister of soda lime to avoid potential cross-contamination [19]. High-efficiency particulate air filters [HEPA] should be used in the anaesthetic machine at the patient's end and between the anaesthetic machine and the expiratory limb of the circuit [14]. The surfaces of electronic appliances coming into frequent contact with staff can be covered with plastic wrap for the ease of decontamination [14].

Measures such as risk assessment and serology testing of members of the teams working in the OR should be done [20]. Additionally, surgical sections should be ideally divided into two categories, a dedicated section for positive patients which only needs minimum OR and surgical staff and another section dedicated for emergency surgery and urgent oncological surgery and depending on the risk assessment, for other routine surgeries in COVID-19 negative patients [21].

Environmental sanitization, proper waste disposal and linen management after every procedure should be carried out adequately. Maintaining hygiene should be the responsibility of all the individuals of the team. Single use contaminated material should be discarded, and reusable material should be decontaminated and disinfected conforming to the institutional protocols. Wearing full PPE is a must during sanitizing procedures. A dedicated area should be assigned for disposal of waste, and appropriate PPE should be worn in transporting and handling such waste. They should not be placed on floors but in dedicated containers and immediately sent for cleaning minimizing the time left outside the OR [17]. The cleaning of surgical equipment used in procedures of a
COVID-19 positive or suspected patient should be done separately from other surgical equipment [22].

\section{Elective surgery during the COVID-19 pandemic}

Due to the impact of the pandemic on the health system, surgeons witnessed a dramatic change in their practice with decreasing elective surgeries at the outset. General surgeons seemed mostly affected by this due to the diverse procedures they perform. Many countries have imposed regulations limiting elective surgeries to make their acute care facilities function at maximum capacity [23, 24]. Despite the frequently changing guidelines, individual decisions should be taken considering the situation in the locality and consulting an expert panel not limited to surgeons but including nurses, resource managers, hospital administration and epidemiologists. Furthermore, it is important to identify the trajectory of the local COVID-19 disease in guiding these recommendations [25].

A joint guideline had been released with the collaboration of the American College of Surgeons, American Society of Anaesthesiologists, Association of Perioperative Registered Nurses, and the American Hospital Association on resuming elective surgery after the COVID-19 pandemic in the USA. The timing of reopening should be after a sustained reduction of the rate of detecting new COVID-19 cases with the hospitals are no longer using crisis standards of care. They also should have adequate staff, ICU beds, PPE, ventilators and other medical supplies. Patient and staff testing should be freely available and proper protocols should be in place for handling positive patients and staff. Prioritization of cases should be done with a scoring system with identification of essential health care staff for the procedures and their willingness to comply with the increased workload. Strategies should be in place for the phased opening of ORs after identifying the capacity goals. Further, the guidance recommends having institutional guidelines for the five phases of surgical care; preoperative, immediate preoperative, intraoperative, postoperative and postdischarge care planning ensuring the safety of patients and staff. It also emphasises on keeping records to reflect and reevaluate. It is further recommended to expect and be ready for a possible second wave of infection and has plans in hand for risk mitigation [26].

\section{Emergency surgery during the COVID-19 pandemic}

Emergency surgical care during the pandemic could be unrelated or directly related to COVID-19. In one hand, emergency surgery may be needed in patients admitted with COVID-19, especially in the ICU they are prone to develop mesenteric ischemia, perforation of hollow viscera and acute limb ischemia [15]. On the other hand, there may be patients with acute surgical problems unrelated to COVID-19 
requiring emergency surgery who have concurrent COVID19 infection [15].

According to the guidelines by the American College of Surgeons, emergency surgical procedures should be undertaken if delaying would likely prolong hospital stay or cause potential harm to the patient. Furthermore, it states that certain surgical conditions may be managed non-operatively or if necessary, in an outpatient setting if possible. These include acute thrombosed haemorrhoids, superficial perianal abscesses, superficial and localised soft tissue infections, uncomplicated appendicitis and symptomatic cholelithiasis. It is advisable to opt for non-surgical interventions [i.e. radiological] in conditions such as acute pancreatitis complicated with necrosis while emergency surgery is mandated in conditions including bowel perforation, intestinal ischemia, and strangulated hernias [27].

Despite the threats posed by the pandemic, trauma patients will require urgent life-saving interventions. This pandemic has hindered the ability of trauma care centres to provide care for the critically injured. Guidelines have been issued on the management for trauma centres during the pandemic. The American College of Surgeons Committee on trauma had proposed a planning process which involves regional planning, hospital planning involving the considerations on ICU capacity and capabilities and training of staff ensuring the protection of both staff and patients.

Furthermore, they recommend strategies that should be implemented at the point of care. The trauma bay should determine a patient's COVID-19 status by an assessment of their symptoms and, droplet and contact precautions should be followed for all patients. The OR should work based on the hospital policy for COVID-19 patients preventing any delays. The hospital should be aware of its ICU capacity and the availability of ventilators. Proper management of limited resources such as blood products and PPE should be done, and hospital policies should be implemented in preserving these. Setting up restrictive transfusion strategies in the ICUs for blood products and encouraging blood donation in the community should be done to conserve and ensure the availability of blood [28].

\section{Conclusions and Recommendations}

The COVID-19 pandemic had caused a paradigm shift in the health sector. It has made a dramatic impact on the professional commitments of surgeons in their diverse specialities. Preventing infection in a high-risk work setting and providing optimum care to the patients by managing limited available resources in the facility is challenging. To face this challenge, modifications in infrastructure, strict healthcare policy changes and solid infection control strategies need to be implemented. This should be done with the involvement of multiple stakeholders including surgeons, anaesthetists, nurses and support staff. Aligning interests of all parties is essential in providing unhindered care at the patients' best interest minimising the risk of viral transmission to other patients and healthcare workers.

\section{Conflicts of interest}

In compliance with the ICMJE uniform disclosure form, all authors declare the following: Payment/services info: All authors have declared that no financial support was received from any organization for the submitted work. Financial relationships: All authors have declared that they have no financial relationships at present or within the previous three years with any organizations that might have an interest in the submitted work.

\section{Other relationships}

All authors have declared that there are no other relationships or activities that could appear to have influenced the submitted work.

All authors disclose no conflict of interest. The study was conducted in accordance with the ethical standards of the relevant institutional or national ethics committee and the Helsinki Declaration of 1975, as revised in 2000 .

\section{References}

1. Borges do Nascimento IJ, Cacic N, Abdulazeem HM. Novel Coronavirus Infection [COVID-19] in Humans: A Scoping Review and Meta-Analysis. 2020;9[4].

2. World Health Organization. Coronavirus disease [COVID-2019] situation reports 2020 [Available from:

https://www.who.int/emergencies/diseases/novel-coronavirus2019/situation-reports.

3. American College of Surgeons. COVID-19: guidance for triage of non-emergent surgical procedures 2020 [Available from: https://www.facs.org/covid-19/clinical-guidance/triage.

4. Royal College of Surgeons of England. COVID-19: Good Practice for Surgeons and Surgical Teams 2020 [Available from: https://www.rcseng.ac.uk/standards-and-research/standardsand-guidance/good-practice-guides/coronavirus/covid-19good-practice-for-surgeons-and-surgical-teams/.

5. American College of Surgeons. Other PPE recommendations. 2020 [Available from: https://www.facs.org/covid19/ppe/additional.

6. Centers for Disease Control and Prevention. Interim Infection Prevention and Control Recommendations for Patients with Suspected or Confirmed Coronavirus Disease 2019 [COVID-19] in Healthcare Settings 2020

[Available from:

https://www.cdc.gov/coronavirus/2019-ncov/infectioncontrol/control-recommendations.html?CDC_AA_refVal =https $\% 3 \mathrm{~A} \% 2 \mathrm{~F} \% 2 \mathrm{Fwww} . c d c$. gov $\% 2 \mathrm{~F}$ coronavirus $\% 2 \mathrm{~F} 2019$ - 
ncov\%2Fhcp $\% 2$ Finfection-control.html.

7. American Society of Anesthesiologists. UPDATE: The Use of Personal Protective Equipment by Anesthesia Professionals during the COVID-19 Pandemic 2020

[Available from: https://www.asahq.org/about-asa/newsroom/ news-releases/2020/03/update-the-use-of-personal-protectiveequipment-by-anesthesia-professionals-during-the-covid-19pandemic.

8. World Health Organization. WHO Director-General's opening remarks at the media briefing on COVID-19 - 16 March 2020 [Available from:

https://www.who.int/dg/speeches/detail/who-director-general-sopening-remarks-at-the-media-briefing-on-covid-19---16march-2020.

9. Al-Muharraqi M. Testing recommendation for COVID-19 [SARS-CoV-2] in patients planned for surgery-continuing the service and 'suppressing'the pandemic. The British Journal of Oral \& Maxillofacial Surgery. 2020.

10.Forrester JD, Nassar AK, Maggio PM, Hawn MT. Precautions for operating room team members during the COVID-19 pandemic. Journal of the American College of Surgeons. 2020.

11.Chiu PWY, Ng SC, Inoue H, Reddy DN, Hu EL, Cho JY, et al. Practice of endoscopy during COVID-19 pandemic: position statements of the Asian Pacific Society for Digestive Endoscopy [APSDE-COVID statements]. Gut. 2020;69[6]:991-6.

12.Rajan N, Joshi GP. COVID-19: Role of Ambulatory Surgery Facilities in This Global Pandemic. Anesthesia and Analgesia. 2020.

13.Centers for Disease Control and Prevention. Social Distancing 2020 [Available from:

https://www.cdc.gov/coronavirus/2019-ncov/prevent-gettingsick/social-distancing.html.

14.Wong J, Goh QY, Tan Z, Lie SA, Tay YC, Ng SY, et al. Preparing for a COVID-19 pandemic: a review of operating room outbreak response measures in a large tertiary hospital in Singapore. Canadian Journal of Anesthesia/Journal canadien d'anesthésie. 2020:1-14

15.Collaborative $\mathrm{C}$. Global guidance for surgical care during the COVID-19 pandemic. Br J Surg. 2020.

16.Brindle ME, Gawande A. Managing COVID-19 in surgical systems. Annals of Surgery. 2020.

\section{List of abbreviations}

COVID-19 : Coronavirus disease-2019

SARS-CoV-2 : Severe acute respiratory syndrome-coronavirus-2

WHO : World Health Organization

PHEIC : Public Health Emergency of Intemational Concem

CDC : Centres for Disease Control and Prevention

RT-PCR : Reverse Transcription -PCR Polymerase Chain Reaction

OR : Operating Room

HEPA : High-efficiency particulate air filters

PAPR : Powered Air Purifying Respirator

PPE : Personal Protective Equipment

ICU : Intensive Care Unit
17.Coccolini F, Perrone G, Chiarugi M, Di Marzo F, Ansaloni L, Scandroglio I, et al. Surgery in COVID-19 patients: operational directives. World Journal of Emergency Surgery. 2020;15:1-7.

18.Centers for Disease Control and Prevention. Sequence for putting on personal protective eqipment 2020 [Available from: https://www.cdc.gov/hai/pdfs/ppe/PPE-Sequence.pdf.

19.Tan T. How severe acute respiratory syndrome [SARS] affected the Department of Anaesthesia at Singapore General Hospital. Anaesthesia and intensive care. 2004;32 [3]:394-400.

20.Brücher BL, Nigri G, Tinelli A, Lapeña JFF, Espin-Basany E, Macri P, et al. COVID-19: Pandemic surgery guidance. 4open. 2020;3:1.

21.Zheng MH, Boni L, Fingerhut A. Minimally invasive surgery and the novel coronavirus outbreak: lessons learned in China and Italy. Annals of surgery. 2020.

22. Society of American gastrointestinal and endoscopic surgeons [SAGES]. SAGES AND EAES RECOMMENDATIONS REGARDING SURGICAL RESPONSE TO COVID-19 CRISIS 2020 [Available from: https://www.sages.org/recommendationssurgical-response-covid-19/.

23.Iacobucci G. Covid-19: all non-urgent elective surgery is suspended for at least three months in England. British Medical Journal Publishing Group; 2020.

24. State guidance on elective surgeries 2020 [Available from: https://www.ascassociation.org/covid-19-state.

25.Diaz A, Sarac BA, Schoenbrunner AR, Janis JE, Pawlik TM. Elective surgery in the time of COVID-19. Elsevier; 2020.

26.American College of Surgeons. Joint Statement: Roadmap for Resuming Elective Surgery after COVID-19 Pandemic 2020 [Available from: https://www.facs.org/covid-19/clinicalguidance/roadmap-elective-surgery.

27.American College of Surgeons. COVID-19: Elective Case Triage Guidelines for Surgical Care 2020 [Available from: https://www.facs.org/covid-19/clinical-guidance/elective-case.

28.American College of Surgeons Committee on Trauma. Maintaining Trauma Center Access \& Care during the COVID19 Pandemic: Guidance Document for Trauma Medical Directors 2020 [Available from: https://www.facs.org/qualityprograms/trauma/maintaining-access.

29.Jayasinghe R, Jayarajah U, Seneviratne S. Consensus in Surgical Practice during the COVID-19 Pandemic: An Appraisal of the Literature. 2020. https://doi.org/10.3332/ecancer.2020.1026

\section{ANZHPBA : Australian and New Zealand Hepatic, Pancreatic and Biliary Association}

HIPPA : Health Insurance Portability and Accountability act

NICE : National Institute for Health and Care Excellence

NHS : National Health Services

ESMO : European Society of Medical Oncology

SAGES : Society of American Gastrointestinal and Endoscopic Surgeons

FFP3 : Filtering Face Piece-3

USA : United States of America

UK : United Kingdom 